\title{
EPSTEIN, Edward; PION-BERLIN, David (eds). 2006. Broken Promises? The Argentine Crisis and Argentine Democracy. Lanham: Lexington Books, 288 pp.
}

María Cosette GodoY ${ }^{1}$

A fines de 2001, Argentina sufrió una de las peores crisis -política, social y económica- que haya experimentado desde el retorno de la democracia en 1983. Las causas, los principales actores, y especialmente sus efectos en el actual sistema democrático, son analizados a lo largo del libro editado por Edward Epstein y David Pion-Berlin². El trance argentino fue producto de un proceso complejo que se pretende examinar desde la mirada comprensiva de diversos autores, enfocada en sus aspectos más relevantes. Los doce capítulos que componen el libro son agrupados en cinco partes que, luego de una introducción, dan cuenta de las causas de la crisis, las respuestas de los ciudadanos, el rol jugado por las fuerzas de orden, las reacciones estatales y de los entes extranjeros, el proceso de reforma institucional, así como de los niveles de apoyo de algunos países como Brasil y los Estados Unidos.

La meta del libro consiste en revisar el proceso argentino de los últimos años, desde diferentes disciplinas de las ciencias sociales y políticas. De ello surgen enfoques metodológicos también divergentes, desde el uso de fuentes secundarias, hasta técnicas de investigación participante. El texto se inscribe en una tradición del pensamiento de izquierda, desde los comentarios de Engels a la Comuna de París de 1871, hasta la más contemporánea revisión de ese pensamiento, a cargo de Hardt y Negri ${ }^{3}$.

1. Licenciada en Ciencias Jurídicas y Sociales, Universidad de Chile. Magíster en Ciencia Política, Instituto de Asuntos Públicos, Universidad de Chile. Correo electrónico: mcosette@gmail.com

2. Edward Epstein es profesor de Ciencia Política en la Universidad de Utah. Por su parte, David Pion-Berlin es profesor de Ciencia Política en la Universidad de California.

3. Nos referimos en especial a la introducción a la obra de Karl Marx, La Guerra Civil en Francia, redactada por Federico Engels, en 1891. La frase tópica es: "Mirad a la Comuna de París: ¡he ahí la dictadura del proletariado!”. En cuanto a Michael Hardt y Antonio Negri, 
La idea de una demanda de cambio desde la base, que se enfrenta tarde o temprano a las cuestiones del orden institucional, fluye de sus diferentes capítulos. En efecto, las instituciones democráticas son puestas en cuestión, pero los requerimientos de una democracia representativa son, a la vez, reinstalados en el curso de la misma crisis. Esta última, al parecer, ventiló los vicios de esa democracia, aun cuando su epílogo parece incierto.

Para los editores, el título Broken Promises?, busca llamar la atención sobre quizás sea el efecto más serio de esta crisis: la declinación de los niveles de confianza pública en las instituciones democráticas fundamentales. Como advierten Epstein y Pion-Berlin, dicha característica se hizo patente en el rechazo ciudadano al cuerpo político en su conjunto a través de la expresión popular "que se vayan todos". Similar rechazo pudo advertirse respecto de los miembros del Poder Judicial y de las fuerzas policiales. A pesar de tal nivel de descrédito, llama la atención el esfuerzo demostrado por esta misma clase política, para buscar una salida institucional sin requerir de la intervención del poder militar. Finalmente, luego de un breve análisis de las medidas adoptadas por el gobierno de Eduardo Duhalde (2002-2003) y de la actual administración de Ernesto Kirchner (2003-2007) para superar el estado de crisis, los autores concluyen que el país actualmente se encuentra en un período de postcrisis, sin que sea evidente cuál será la extensión y orientación que tendrá este período.

Una de las primeras preguntas que surge de la situación vivida en Argentina a fines de 2001, está relacionada con la identificación de sus causas. En la segunda parte del libro, tres diferentes autores nos brindan su análisis al respecto. En un primer artículo, José Nun ${ }^{4}$ revisa las dificultades presentes en el proceso democrático argentino. Es sugerente en su texto el uso de la metáfora de Mancur Olson, quien distingue entre "bandidos vagabundos" y "bandidos estacionarios". La diferencia entre ambos se basa en los compromisos que adquieren unos y otros con el espacio en el cual efectúan sus incursiones; para los primeros, la apropiación de los beneficios se logra por la apropiación de todo lo que encuentran a su paso; para los segundos, la clave está en dejar a sus víctimas lo suficiente para que puedan seguir produciendo y, con ello, continuar su explotación. Tal distinción permite a Nun profundizar en su hipótesis acerca de la crisis de gobernabilidad en la democracia representativa provocada por la posición que adquieren grupos privilegiados,

nos referimos en particular su texto Multitude. War and Democracy in the Age of Empire, New York: Penguin Press, 2004, donde los autores deconstruyen la identidad de las izquierdas y su referencia a los modelos de orden estatal.

4. El autor es abogado y cientista político, actual Secretario de Cultura de la Presidencia de la Nación argentina. Ha escrito diversos artículos sobre la situación política argentina y latinoamericana. 
particularmente grandes empresarios y grupos económicos, en un esquema económico de tipo rentista, excesivamente concentrado y excluyente. La búsqueda de inversiones provenientes del exterior, según muestra el autor, se efectuó sobre la base de una apertura al capital de corto plazo, promoviendo un ciclo de hegemonía del capital financiero que logró una excesiva participación en los asuntos públicos. Radicado en la experiencia dictatorial, este ciclo se habría extendido a la restauración democrática del presidente Alfonsín (1983-1989), presentándose luego en el neopopulismo conservador menemista (1989-1999) y en la posterior ruptura ocurrida en la presidencia de De la Rúa (1999-2001). En este período, la lucha por el control de las instituciones se enfrentó además a los procesos desregulatorios reclamados por los grupos asociados al capital financiero, los que, en definitiva, devinieron en nuevas regulaciones a favor de dichos grupos de interés. El resultado fue una excesiva concentración de la economía y un incremento ostensible de la pobreza, la erosión de la red de seguridad social, el debilitamiento de los derechos ciudadanos y de las instituciones que los garantizaban.

El texto de Nun enlaza con el de Marcelo Fabián Sain ${ }^{5}$, quien aborda la cuestión de la policía, la política y la sociedad civil en la provincia de Buenos Aires. Para Sain, la provincia de Buenos Aires es un caso de Estado fallido: la policía aparece como un cuerpo ligado a los objetivos políticos del gobierno de la provincia, más que a la protección de los derechos y libertades de sus ciudadanos. Los efectos en el tratamiento de la criminalidad son también analizados, en particular el control de los actos criminales que se realizan desde la policía y la manera en que ésta terminó involucrándose en algunos hechos delictivos. La crisis institucional y social de Argentina se ve expresada también en la aparición de una mayor y nueva criminalidad en Buenos Aires, particularmente en los barrios pobres, donde no existe un efectivo control estatal. Tanto el tráfico organizado de drogas y armas, como el negocio del secuestro, habrían contado con protección policial. La débil experiencia del Estado en el control de lo ilícito se enfrenta por una parte con estas nuevas formas de criminalidad, toleradas, asistidas o desarrolladas directamente por miembros de la policía y, por otra, con la reticencia del aparato policial a someterse a transformaciones institucionales. Para el autor, esta cuestión no se sitúa en el marco de los desafíos culturales, sino precisamente en la necesidad de una modernización institucional de la policía como uno de los requisitos para la consolidación democrática.

Jorge Schvarzer6, en el último artículo de esta segunda parte, vuelve, a su vez, a tratar los efectos de la hegemonía financiera sobre los aspectos

5. El autor es cientista político y profesor de la Universidad Nacional de Quilmes. Ha escrito sobre relaciones entre civiles y militares, así como seguridad pública.

6. Economista e investigador de la Universidad de Buenos Aires. Es autor de libros y artículos acerca de la economía argentina. 
sociales y económicos de la vida argentina, producto del Plan de Convertibilidad (1991-2001). Examina las estrategias de este plan, consistentes, por una parte, en el establecimiento de una tasa fija de cambio entre el peso y el dólar y, por otra, en la suspensión de la emisión de moneda, medidas destinadas a mantener el equilibrio de la masa monetaria. Las consecuencias del plan son revisadas en el ámbito de la producción nacional y del consiguiente incremento en las actividades primarias y la dotación de servicios. La contrapartida habría estado en la generación de un déficit estructural en la balanza comercial y en la contracción del empleo industrial. De esta manera, dicho programa tuvo como resultado un cambio en la distribución de los ingresos, favoreciendo a los estratos con mayores dotaciones de recursos, en desmedro de los sectores más pobres. A ello se habrían sumado efectos negativos sobre el empleo y los ingresos, como consecuencia del mismo déficit estructural, que debió ser compensado mediante la apertura al capital extranjero. La expansión del acceso al capital extranjero supuso presiones sobre la repatriación de dividendos e intereses hacia el exterior, agravándose el problema en un verdadero círculo vicioso. Las soluciones se enfocaron en mantener un balance positivo, al menos en algunas áreas de las exportaciones, cuestión que se vio frustrada por la devaluación brasileña, la caída en el precio de las materias primas y la sobrevaluación internacional del dólar, que se encontraba atado al peso argentino. Las consecuencias en el presupuesto público, así como en el sistema financiero y monetario, son también examinadas en este capítulo. Se evalúan además las reacciones ante la crisis, dando cuenta de cómo la política económica argentina se apartó progresivamente del esquema de convertibilidad. La crisis del año 2001 es analizada, finalmente, a través de sus consecuencias institucionales y políticas: cinco presidentes en diez días.

La respuesta ciudadana a la crisis es tratada en la tercera parte del libro. En el primero de los artículos de esta parte, Edward Epstein examina el movimiento de piqueteros en el Gran Buenos Aires. La cuestión que atraviesa el análisis dice relación con la emergencia de un tipo particular de acción colectiva, representado por los pobres desempleados en el marco de la crisis trasandina. Para el autor, ésta tiene efectos en los vínculos sociales tradicionales de membresía en el movimiento sindical o en las redes de clientelismo vecinal. La posibilidad que de este movimiento surjan nuevas formas de clientelismo es planteada en el texto, a partir de la vinculación de los liderazgos piqueteros con el aparato político. Los programas de asistencia social son, precisamente, el ámbito en que aquella posibilidad parecería plantearse. Sin embargo, las características de la acción colectiva definen tipologías de la negociación, entre segmentos duros y dialogantes, lo que daría cuenta de una tradición ya definida en el movimiento sindical del tipo Confederación General del Trabajo (CGT). La cultura piquetera es examinada a partir del análisis de las condiciones del liderazgo y de las derivas que se producen, debido a 
las modificaciones que sufre el entorno del movimiento. El control del orden público aparece, entonces, como una cuestión central en esas derivas, particularmente cuando se trata de poner a prueba el liderazgo estatal en el gobierno de Kirchner.

Por su parte, Maristella Svampa ${ }^{7}$ y Damián Corral $^{8}$ se adentran en otro aspecto de la respuesta colectiva ciudadana: las asambleas vecinales. Examinan los casos de Villa Crespo y Palermo. Los autores describen estas experiencias como un espacio para la organización y la deliberación. Lo destacable es que ellas pretendieron constituirse en nuevas formas y medios de representación política, en un escenario crítico del enfoque neoliberal. En las asambleas vecinales se logra el contacto entre distintas texturas del espacio social, aunque con una fuerte presencia de profesionales de la clase media. Se examina en el texto el trasfondo de la fragmentación de este tipo de experiencias. Destaca cómo el estudio de tales asambleas expresa una demanda por más autonomía, como una forma contracultural de la propia acción colectiva. No se trata de una simple transposición del esquema de representación política, ni siquiera de sus formas simbólicas. De hecho, en la primera etapa de estas asambleas vecinales se optó por expresiones de acción directa junto a representaciones culturales callejeras. Sin embargo, esta misma forma fue rápidamente criticada por los partidos de izquierda y por el conocimiento experto de intelectuales y profesionales, conduciendo a una rápida desintegración de este nuevo espacio democrático. La cuestión se presentó entonces de manera doble, primero en las aspiraciones autonomistas de las asambleas, y luego en su subordinación política proveniente del medio en que ellas se desenvolvían. Esto se manifestó en fuertes disensiones al interior de las asambleas, las cuales terminaron sus días hacia fines de 2003. Con todo, ellas pusieron en el orden del día una apuesta contracultural, de autonomía respecto de los partidos, de los sindicatos y del Estado. Esa demanda es para los autores un tópico común de los movimientos sociales desde los años 1990.

Luego, Inés González Bombal ${ }^{9}$ y Mariana Luzzi ${ }^{10}$ exponen la experiencia de los clubes de trueque, como alternativas de mercado, donde los principios de solidaridad y ayuda mutua buscaron reemplazar la competencia en la búsqueda de beneficios. Ellos proporcionaron la posibilidad de que sectores empobrecidos pudieran adquirir bienes sin necesidad de pago en moneda efectiva, dada la fuerte caída en los ingresos de esos sectores como consecuencia de la crisis económica. El esquema funciona-

7. La autora es socióloga, investigadora-docente de la Universidad Nacional de General Sarmiento e investigadora del CONICET.

8. Docente e investigador de la Universidad Nacional de General Sarmiento.

9. Socióloga, investigadora del Centro de Estudios de Estado y Sociedad (CEDES) y docente en la Universidad Nacional de General Sarmiento.

10. Socióloga, investigadora docente de la Universidad Nacional de General Sarmiento. 
ba mediante la emisión de créditos que llegaron a conocerse como "dinero social" y que servían para ajustar los precios de lo transado dentro de los clubes. Lo rescatable para las autoras se encuentra relacionado con la experiencia organizativa de esos clubes, en el espacio de las clases medias. Destacan también su extensión a los sectores pobres y su condición de nodos a través de los cuales los usuarios avanzan hacia la satisfacción de sus necesidades. En ese sentido, constituyen estrategias de supervivencia más que proyectos comunitarios, donde el liderazgo se manifiesta como capacidad de coordinación de las necesidades y las ofertas de satisfacción, y no solamente como escenarios para la movilización colectiva. Dos principios rigen este tipo de organizaciones; el primero, que cada miembro del club dispone de dos roles, uno de productor y otro de consumidor; el segundo, que la función básica de estos clubes consiste en la creación de nuevos espacios para el intercambio, donde todos los participantes se consideran iguales. El doble rol de los actores lleva a la creación de un neologismo, el de prosumidor que, para las autoras, involucraba también la posibilidad de desplegar opciones de desarrollo personal, dado que los clubes de trueque permitían ofrecer habilidades capaces de ser traducidas en la provisión de bienes y servicios. El texto da cuenta de la crisis de los clubes de trueque cuando los bienes y servicios ofrecidos en ellos debieron enfrentar el alza de precios dentro del sistema económico argentino y la emisión no controlada de créditos dentro de los clubes. El ajuste se habría hecho mediante una combinación de créditos y dinero, a partir del año 2002, lo cual trajo como consecuencia una división en los clubes de trueque, entre aquellos participantes que disponían de recursos, dinero y alimentos, y quienes sólo podían disponer de bienes de segunda mano. Esta situación quebró el principio de evitar el dinero como medio de cambio y produjo desconfianza en el crédito o dinero social. Esto impactó en la propia estructura de los clubes de trueque, en la medida que ellos se habían caracterizado más bien por tratarse de lugares donde se transaban bienes y servicios, que por constituir referentes de identificación. En definitiva, no se trataba de proyectos comunitarios, sino más bien del ofrecimiento de un espacio para el desarrollo de estrategias individuales de supervivencia.

La cuarta parte del libro analiza el papel desempeñado por las fuerzas de orden durante los sucesos de fines de 2001, por medio de dos artículos relativos a las Fuerzas Armadas (FFAA) y al cuerpo de Gendarmería, respectivamente. La decisión de las FFAA de restarse a participar en las instancias de solución a la crisis, es abordada por Rut Diamint ${ }^{11}$, a través del cuestionamiento del proceso de institucionalización de las relaciones entre poder político y poder militar. Si bien la actitud de las FFAA constituyó para muchos una prueba de su subordinación a la autoridad civil,

11. Socióloga y profesora de la Universidad Torcuato Di Tella; es autora de artículos en revistas y libros sobre seguridad regional y hemisférica. 
el análisis más detallado contenido en este artículo muestra las tensiones que todavía existen entre ambos poderes. La ausencia de una política de defensa -o más precisamente, la reproducción por las FFAA y de seguridad, de doctrinas y comportamientos vigentes desde su creación-, los juicios pendientes por violaciones a los derechos humanos y los problemas surgidos a raíz del rol constitucional del Congreso en la aprobación de las promociones militares, revelan que el control democrático sobre las Fuerzas Armadas aún no se encuentra consolidado.

Respecto a la actuación de la Gendarmería Nacional Argentina, resulta novedoso el enfoque utilizado por Eric Stener Carlson ${ }^{12}$. A pesar de los cuestionamientos surgidos por la muerte de cuatro personas en los disturbios que tuvieron lugar en Corrientes (1999) y en Salta (2001), y que revivieron críticas por las muertes y actos de tortura que tuvieron lugar durante el período de la Guerra Sucia (1976-1982), las entrevistas realizadas a algunos gendarmes reflejaron su empatía con las protestas sociales. $Y$ aunque ellos se describen a sí mismos como funcionarios y representantes del poder del Estado, sus magras condiciones de vida y la coexistencia con quienes protestaron y se enfrentaron con ellos, han tenido como efecto el surgimiento de sentimientos de identificación y la percepción de su condición de trabajadores pobres con uniforme.

Finalmente, la última parte del libro hace referencia al proceso político argentino, así como a las respuestas estatales y extranjeras a la crisis. En el primer artículo de esta sección, Isidoro Cheresky ${ }^{13}$ pone el acento en las transformaciones que tuvieron lugar en la vida política argentina como consecuencia de la crisis. La desafección ciudadana respecto de los partidos políticos puso en tela de juicio la estructura política tradicional. Por otra parte, el liderazgo del presidente Kirchner sustentado en el apoyo ciudadano demostró eficiencia en el logro de cambios políticos significativos. Como lo precisa el autor, un gobierno personalista basado en la opinión pública, puede lograr amplia libertad de acción en el corto plazo. Aunque, como contrapartida, esta característica eventualmente podría debilitar el liderazgo de Kirchner, al prescindir éste de las estructuras organizacionales mínimas que le brindan estabilidad al conjunto del sistema institucional.

El segundo capítulo constituye un interesante aporte efectuado por Juan Abal Medina ${ }^{14}$ acerca de la crisis política y de las reformas que deben llevarse a cabo en el corto plazo. El autor describe cómo se fue gestando el proceso gradual de desencanto, desafección y posterior enojo e indignación de la población, a partir de un momento inicial de

12. El autor es un reconocido experto en derechos humanos, habiendo trabajado por muchos años en organismos internacionales, entre ellos el Tribunal Penal Internacional para la ex Yugoslavia y en la identificación de cuerpos de desaparecidos en Argentina.

13. Sociólogo, profesor de la Universidad de Buenos Aires e investigador del CONICET.

14. Cientista político y profesor de la Universidad de Buenos Aires. 
euforia por el retorno de la democracia en 1983. Su reflexión nos permite ir más allá de la experiencia política argentina para situarnos en nuestra propia realidad, al identificar procesos comunes de desafección y desconocimiento de las instancias regulares de representación política, que llevaron a amplios sectores de la sociedad a confrontarse directamente con el Estado, obviando los canales formales de procesamiento de las demandas sociales.

Por último, en el tercer artículo, Roberto Russell ${ }^{15}$ y Juan Gabriel Tokatlian $^{16}$ describen la evolución de la política internacional argentina respecto a sus dos aliados más importantes de la región: Estados Unidos y Brasil. Tales relaciones han estado marcadas por la competencia con Brasil por la hegemonía en la región y por momentos de subordinación y de acercamiento de tipo estratégico con los Estados Unidos. Los autores abordan también la actitud de ambos países durante la crisis y los desafíos del actual gobierno en la definición de una política exterior consistente, la cual debiera estar dirigida al establecimiento de relaciones de integración que dejen de lado confrontaciones de tipo ideológico y pretensiones de carácter hegemónico.

Como es posible advertir, Broken Promises? brinda al lector un análisis acabado que trasciende el examen de las causas de la crisis argentina de fines de 2001. Su lectura permite comprender la complejidad de dicho proceso a través del tratamiento exhaustivo del conjunto de los actores involucrados, tanto estatales como sociales, quienes se han visto enfrentados al desafío de participar en la reconstrucción de un sistema político democrático cuyas bases institucionales se encuentran aún fuertemente cuestionadas.

Por otra parte, y desde una mirada práctica, el libro editado por Edward Epstein y David Pion-Berlin, constituye una valiosa herramienta para el estudio del proceso político argentino, al contener la voz autorizada de diversos especialistas. Sin embargo, se echa de menos en este trabajo compilatorio una mayor preocupación en la edición del mismo, cuestión que queda reflejada en la ausencia de referencias bibliográficas de los autores así como en la falta de capítulos introductorios en cada una de las cinco partes que lo componen. En efecto, el aporte de cada uno de los autores adquiere más bien la forma de artículos de revista que de capítulos de libro. No obstante, esta crítica -meramente formal- no le resta mérito al contenido del mismo.

15. Abogado, profesor de la Universidad Nacional de Tucumán. Coordinador Académico del Instituto del Servicio Exterior de la Nación del Ministerio de Relaciones Exteriores, Comercio Internacional y Culto de Argentina. Es también profesor de la Universidad Torcuato Di Tella y autor de diversas publicaciones en el área de las relaciones internacionales.

16. Especialista en relaciones internacionales y profesor de la Universidad de San Andrés. 